\title{
UNDERSTANDING OF CROP LODGING INDUCED CHANGES IN SCATTERING MECHANISMS USING RADARSAT-2 AND SENTINEL-1 DERIVED METRICS
}

\author{
S. Chauhan ${ }^{1, *}$, R. Darvishzadeh ${ }^{1}$, M. Boschetti ${ }^{2}$, A. Nelson ${ }^{1}$, \\ ${ }^{1}$ Faculty of Geo-information Science and Earth Observation (ITC), University of Twente, Enschede 7500AE, The Netherlands - \\ (s.chauhan, r.darvish, a.nelson)@utwente.nl \\ ${ }^{2}$ CNR-IREA, Institute for Electromagnetic Sensing of the Environment, National Research Council, 20133 Milano, Italy - \\ boschetti.m@irea.cnr.it
}

KEY WORDS: Crop lodging, RADARSAT-2, Sentinel-1, H/ $\alpha$ Wishart classification, Sustainable agriculture

\begin{abstract}
:
Crop lodging - the bending of crop stems from the vertical - is a major yield-reducing factor in cereal crops and causes deterioration in grain quality. Accurate assessment of crop lodging is important for improving estimates of crop yield losses, informing insurance loss adjusters and influencing management decisions for subsequent seasons. The role of remote sensing data, particularly synthetic aperture radar (SAR) data has been emphasized in the recent literature for crop lodging assessment. However, the effect of lodging on SAR scattering mechanisms is still unknown. Therefore, this research aims to understand the possible change in scattering mechanisms due to lodging by investigating SAR image pairs before and after lodging. We conducted the study in 26 wheat fields in the Bonifiche Ferraresi farm, located in Jolanda di Savoia, Ferrara, Italy. We measured temporal crop biophysical (e.g. crop angle) parameters and acquired multi-incidence angle RADARSAT-2 (R-2 FQ8-27 and R-2 FQ21-41 ${ }^{\circ}$ ) and Sentinel-1 (S-1 $40^{\circ}$ ) images corresponding to the time of field observations. We extracted metrics of SAR scattering mechanisms from RADARSAT-2 and Sentinel-1 image pairs in different zones using the unsupervised $\mathrm{H} / \alpha$ decomposition algorithm and Wishart classifier. Contrasting results were obtained at different incidence angles. Bragg surface scattering increased in the case of S-1 (6.8\%), R-2 FQ8 (1.8\%) while at R-2 FQ21, it decreased (8\%) after lodging. The change in double bounce scattering was more prominent at low incidence angle. These observations can guide future use of SAR-based information for operational crop lodging assessment in particular, and sustainable agriculture in general.
\end{abstract}

\section{INTRODUCTION}

Crop lodging - the permanent displacement of the crop stem from its upright position or destruction of root anchorage is caused by the complex interaction between genetic, management and environmental forces (Pinthus, 1974). Lodging is a common phenomenon in wheat and is a major constraint to increase wheat yield (Peng et al., 2014). Furthermore, studies show that lodging results in deterioration of grain quality, reduction of photosynthetic activity, slowed harvest and increased drying costs (Berry et al., 2004). Hence, scientific understanding of how remote sensing (RS) signals change due to lodging can be useful for crop inventories, boosting productivity and enabling efficient use of resources.

Field-based approaches for lodging assessment rely on visual inspection and physical measurement of plant properties (such as crop angle and lodged area) which can be infeasible for large areas (more than a few 100 ha). Furthermore, these methods depend on the skill and self-consistency of the observer. Alternatively, RS is a cost-effective and scalable approach for obtaining crop lodging information across vast areas. However, the use of RS has focused on detecting crop lodging in individual fields using airborne data rather than at regional scales. For instance, the earliest work by Gerten and Wiese (1987) captured aerial photos of a single wheat field to determine if lodged areas could be discriminated and measured from RGB and near-infrared (NIR) photos. In another study, Chapman et al. (2014) conducted field experiments on 3 ha wheat fields to quantify lodged areas using aerial RGB and NIR images. Yang et al. (2017) used UAV data, a digital surface model (DSM) and textural features to classify lodging in a 306 ha rice field.

The potential of spaceborne synthetic aperture radar (SAR) data has been emphasized in the literature for crop lodging assessment over large and diverse areas. Yang et al. (2015) and Chen et al. (2016) investigated the temporal variation of the features derived from fully polarimetric RADARSAT-2 data to understand their sensitivity to lodging in wheat and sugarcane, respectively. Chauhan et al. (2020a) developed a quantitative approach to detect crop lodging stages (moderate, severe and very severe) based on the crop angle of inclination estimated using Sentinel-1 and RADARSAT-2. A similar study by Shu et al. (2019) also highlighted the role of Sentinel-1 SAR data in classifying lodging stages.

Despite existing SAR-based approaches to detect and classify lodging, the effect of lodging on SAR scattering mechanisms has not been explored. Such knowledge is important to identify metrics that can be used in algorithms for lodging detection. Many target decomposition algorithms such as the incoherent scattering model (Lee et al., 2013) and generalized double and odd-bounce scattering models (Chen et al., 2013) have been developed to extract quantitative parameters related to physical scattering mechanisms. Among them, the $\mathrm{H}-\alpha$ decomposition algorithm (Cloude and Pottier, 1997) combined with Wishart classifier (Lee et al., 1999) provides a simple and effective way of quantifying the important scattering mechanisms from the crops (Guo et al., 2018).

\footnotetext{
* Corresponding author
} 
In this study, we aim to understand the change in scattering mechanisms before and after lodging using combined $\mathrm{H}-\alpha$ and Wishart classifier. This is achieved by comparing the outputs of multi-incidence angle RADARSAT-2 (R-2 FQ8-27 and R-2 FQ21-41 ${ }^{\circ}$ and Sentinel-1 (S-1) data. The results are comparable since near date images are considered from both sensors (Łoś et al., 2016).

\section{DATA ACQUISITION}

\subsection{Study area}

We carried out the study in the Bonifiche Ferraresi farm located


Italy (Fig. 1). The durum and soft wheat cultivars were sown in 26 fields covering 600 ha of the total farm area (3850 ha) in Oct-Nov 2017 and were harvested in June 2018. The size of the wheat fields ranged from 2 to 80 ha.

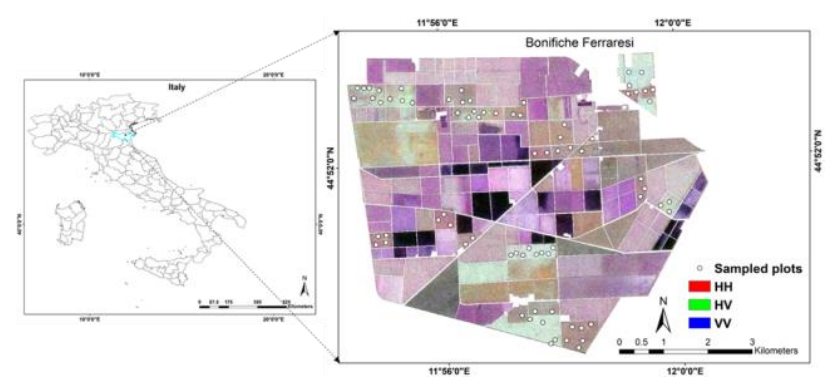

Figure 1. The study area (left) and location of the sampled plots overlaid on a RADARSAT-2 composite (right) acquired on 31 May 2018.

\subsection{Field data}

We established 76 plots $(60 \times 60 \mathrm{~m})$ (Fig. 1) in the wheat fields using a stratified random sample procedure. We measured the percentage of crop lodged area (LA, [0-100]) and crop angle of inclination from the vertical (CAI, [0-90]) in the plots between March and June 2018, in which all the plots were revisited three times, for a total of 228 samples. A lodging score (LS, [0,1]) was then calculated using Eq. 1 .

$$
L S=\frac{C A I^{\circ}}{90^{\circ}} * \frac{L A \%}{100}
$$

The first few instances of lodging were recorded close to the $1^{\text {st }}$ of May 2018. The plots with LS $=0$ were classified as healthy (He, $n=160$ ) while the remaining lodged were categorized as lodged $(\mathrm{L}, n=68)$. The summary statistics are presented in Table 1. In He plots, we identified three subplots $(2 \times 2 \mathrm{~m})$ to carry out the measurements while for L plots, we increased the number of subplots to 4-8 depending on the lodged area (LA).

\begin{tabular}{|c|l|l|c|c|}
\hline Parameter & \multicolumn{2}{|c|}{ Mean } & \multicolumn{2}{c|}{ COV } \\
\hline & He & L & He & L \\
\hline CAI & 2.69 & 50.85 & 0.65 & 0.37 \\
\hline LA & 0.00 & 100.00 & 0.00 & 0.21 \\
\hline LS & 0.000 & 0.884 & 0.00 & 0.45 \\
\hline
\end{tabular}

Table 1. Summary statistics of measured parameters for $\mathrm{He}$ and $\mathrm{L}$ samples. COV is the coefficient of variation.

\subsection{Remote sensing data}

We acquired ten R-2 and 19 S-1 images over the study area between 14 March and 30 June 2018 that were synchronous to the field data measurements. We obtained fine-quad pol (FQ) R2 data from the Canadian Space Agency through the SOAR (Science and Operational Applications Research for RADARSAT-2) program. We selected two-beam modes: steep incidence angle R-2 FQ8 (resampled to $10 \mathrm{~m}$ spatial resolution with $\sim 27^{\circ}$ incidence angle, $25 \times 25 \mathrm{~km}$ swath, ascending mode) and shallow incidence angle R-2 FQ21 (resampled to $7 \mathrm{~m}$ spatial resolution, $\sim 41^{\circ}$ incidence angle, $25 \times 25 \mathrm{~km}$ swath, descending mode). We also acquired S-1 single look complex images in the Interferometric Wide (IW) swath mode with dual polarisation ( $\mathrm{VV}$ and $\mathrm{VH}$ ) from the Copernicus Open Access Hub (resampled to $15 \mathrm{~m}$ spatial resolution with $\sim 40^{\circ}$ incidence angle, $250 \times 250 \mathrm{~km}$ swath and ascending mode).

\section{METHODOLOGY}

\subsection{Data pre-processing}

We pre-processed the R-2 and S-1 images using the Sentinel-1 toolbox in SNAP 6.0. In the case of R-2 data, we first applied the orbit file correction on the images to update the orbit state vectors. The images were then subset for the area of interest, radiometrically calibrated and speckle filtered using a polarimetric Refined Lee speckle filter $(5 \times 5)$. We then terrain corrected the images and masked out the wheat fields. The scattering mechanisms were then investigated (from healthy and lodged fields) from the resultant images of the unsupervised $\mathrm{H}$ $\alpha$ decomposition and Wishart classification.

Due to the acquisition of S-1 data in TOPSAR mode, the processing chain of S-1 data is a bit different than that of R-2. After applying the orbit file correction, we used the TOPSAR split operator to split each sub-swath (IW1, IW2, IW3) and extract the one with our area of interest (IW2). We then calibrated the images and performed a deburst operation to merge the bursts in the azimuth direction and get a seamless image. Just like R-2 data, the images were then subset, speckle filtered, classified, masked and geocoded.

\section{2 $\mathrm{H} / \alpha$ and Wishart Classification}

The unsupervised polarimetric classification combining $\mathrm{H}-\alpha$ decomposition and the Wishart classifier was performed in SNAP to extract the scattering mechanisms. The $\mathrm{H}-\alpha$ decomposition method results in two polarimetric target decomposition parameters, entropy $(\mathrm{H})$ and scattering alpha angle $(\alpha)$, which are derived from the eigenvalue decomposition of the coherency matrix. The $\mathrm{H}[0,1]$ corresponds to the heterogeneity or randomness of the scattering while $\alpha\left[0,90^{\circ}\right]$ corresponds to the variation in scattering mechanisms. The decomposition results form a basis for the initial classification and then the maximum likelihood complex Wishart distribution based classifier reclassifies the image iteratively based on Wishart distances to the cluster centres. The Wishart distance measure from the covariance matrix $(Z)$ to the cluster centre $\left(V_{m}\right)$ of the $m^{\text {th }}$ class is mathematically defined in Eq.2-4.

$$
\begin{aligned}
d\left(Z, V_{m}\right) & =\ln \left|V_{m}\right|+\operatorname{Tr}\left(V_{m}^{-1} Z\right) \\
Z & =\frac{1}{n} \sum_{k=1}^{n} h_{k} h_{k}^{*^{T}} \\
V_{m} & =E\left[Z \mid Z \in \omega_{m}\right]
\end{aligned}
$$


where $\quad h_{k}=$ scattering matrix of $k^{\text {th }}$ sample,

Superscript ' $*$ ' $=$ complex conjugate, $\mathrm{n}=$ number of looks (or samples)

$\omega_{m}=$ pixels belonging to the $m^{\text {th }}$ class

The pixel is assigned to class $\omega_{m}$ if

$$
d\left(Z, V_{m}\right) \leq d\left(Z, V_{j}\right) \text { for all } j \neq m .
$$

All the pixels in the image were classified into nine zones ( $\mathrm{Z} 1$ to Z9) on the $\mathrm{H}-\alpha$ plane, each characterising different scattering mechanisms. Z1-Z3, Z4-Z6 and Z7-Z9 correspond to low, medium and high entropy environments with dihedral reflector (Z1), dipole (volume) (Z2), Bragg surface (Z3), double reflection (Z4), anisotropic particles (Z5), random surface (Z6), complex structures (Z7) and random anisotropic particles (Z8) scattering mechanisms. Z9 is a non-feasible region in the $\mathrm{H}-\alpha$ plane. The classification plane is depicted in Fig. 2. The initial partitioning of the nine classes (eight usable) as shown in this figure has been suggested by Cloude and Pottier (1997). The bounds illustrate that at higher $\mathrm{H}$, the ability to classify various scattering mechanisms is very limited. Detailed explanations regarding different scattering behaviours can be found in Cloude and Pottier (1997) and Lee et al. (1999).

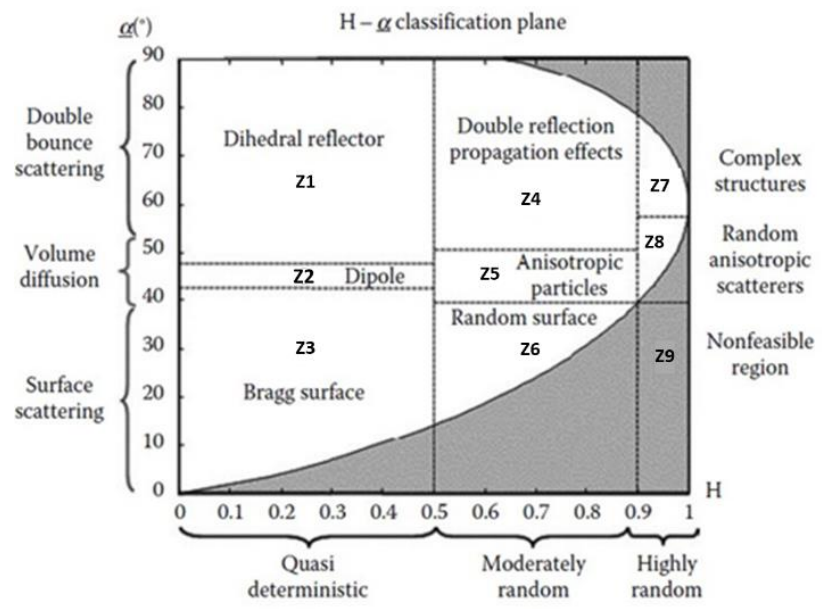

Figure 2. H- $\alpha$ classification plane (Jagdhuber et al., 2014)

\subsection{Data Analysis}

Prior to performing the classification, some experiments with the dense time-series data of Sentinel-1 (March-June 2018) were performed which showed that the first instance of lodging occurred somewhere between the end of April and beginning of May (results not shown) (Chauhan et al., 2020b). Towards the end of the observation period, $80 \%$ of the plots were lodged with varying severities. To understand the possible change in scattering mechanisms, we selected the best possible image pair (before and after lodging) from each dataset. The field observations and the dense time-series analysis showed that before 27 April 2018, all 76 plots were healthy and were at the end of the vegetative growth. The temporal offset between the image pairs of R-2 with respect to S-1 data ranged from 1 day (R-2 FQ8) to the same day (R-2 FQ21) to enable the comparison. The image pairs that were selected for the analysis were: 25 April-12 June (S-1) and 26 April-13 June (R-2 FQ8); 13 April-31 May (S-1) and 13 April-31 May (R-2 FQ21). After classifying the R-2 and S-1 images, the statistics related to each scattering mechanisms were extracted and the differential characteristics of the scattering mechanisms were assessed. The $\mathrm{H}-\alpha$ classification planes (similar to the one showed in Fig. 2) were also extracted for each image to represent the information in the coherency matrix.

\section{RESULTS AND DISCUSSION}

In this section, we utilise the R-2 (multi-incidence) and S-1 datasets to assess the differential characteristics of the scattering mechanisms before and after lodging. The analysis has been divided into two sub-sections. The first one analyses the comparative performance of S-1 and R-2 FQ8 data while the second one compares the results of S-1 and R-2 FQ21 data since studies show that the polarization signature can be influenced by the incidence angle (Jafari et al., 2015).

\subsection{Assessment of scattering mechanisms using S-1 and low incidence angle R-2 FQ8}

The temporal offset between the satellite image pairs of S-1 and R-2 FQ8 data was one day to facilitate the comparison of the two sensors. Fig. 3 depicts the scatter plots for each image in the $\mathrm{H}-\alpha$ classification plane, while the quantitative percentage statistics of the scattering mechanisms from the healthy and lodged plots in each zone are shown in Table 2. Analysis of the distributions of points in the $\mathrm{H}-\alpha$ plane (Fig. 3) and the percentage scattering in Table 3 , reveals that $\mathrm{H}-\alpha$ values changed after lodging incidence. $\mathrm{H}=0$ indicates the presence of a single dominant scattering mechanism, and as it approaches $\mathrm{H}=1$, all scattering mechanisms become dominant. In the case of S-1, the largest densities in the two planes correspond to surface scattering (Z6: $41.01 \%$ before lodging and $39.51 \%$ after) with moderate to high randomness $(\mathrm{H})$. On the other hand, the double bounce scattering (Z4) is non-existent (only 2\% before lodging and $0 \%$ after). For R-2, the largest densities in the two planes correspond to surface scattering (Z6, 25.03\% before and $27.00 \%$ after) and volume diffusion (Z5 12.78\% before $22.19 \%$ after) with moderate to high randomness with some double-bounce scattering at moderate H (Z4 2.29\% before and $0 \%$ after).

The change in the scattering mechanisms was found to be consistent across S-1 and R-2 FQ8 data in most of the zones (Table 2). Previous studies have shown that Bragg scattering (Z3) depends on the incidence angle (Wang et al., 2016), and the same was observed here. The $\mathrm{Z} 3$ scattering corresponding to Bragg surface increased by $6.8 \%$ and $1.8 \%$ (for S-1 and R-2 data respectively) after lodging. On 12 and $13^{\text {th }}$ June, the crop was mature, and the plant water content of wheat leaves had decreased (most leaves had turned yellow and dry). Most of the plots during this time were lodged $\left(\mathrm{LA}=100 \%, \mathrm{CAI}>55^{\circ}\right)$ resulting in the formation of a nearly flat, but rough surface. The change in scattering due to lodging is manifested through the changes in the crop biophysical properties (such as crop height, crop angle, fractional cover, etc.). The medium entropy Z4 scattering, corresponding to the double reflection mechanism, decreased after lodging, with higher contrast in values at a low incidence angle $(11.35 \%$ decrease in R-2 FQ8) as opposed to what we observed in the S-1 data. In the event of lodging, the vertical crop structure is destroyed, causing the dihedral structure (formed by the stem and the ground) to disappear, thus reducing the double-bounce reflection.

The single Z5 scattering, caused by to a cloud of anisotropic particles $\left(\alpha=45^{\circ}\right)$, also increased moderately by $9.4 \%$ in the S-1 data (Table 2) which denotes the moderately random nature of the lodged canopy with high volume scattering. The C-band 
wave can penetrate the wheat canopy more easily when the crop is fully mature; resulting in an increase in scattering caused by the interaction among the wheat stems and a decrease in the surface scattering. Also, direct volume scattering (Z5) from the wheat canopy emerges beyond a $40^{\circ}$ incidence angle (Balenzano et al., 2010). Furthermore, the high entropy Z7 scattering at low incidence angle showed a high increase (10.23\%) after lodging, which can be explained by the complex structures formed by heterogenous lodging occurrence.


The classified S-1 and R-2 image pairs are shown in Fig. 4. The unsupervised classification allowed the identification of various scattering mechanisms and helps discriminate the scattering behaviour of healthy and lodging in general. However, no unique scattering mechanism could be associated with different lodging severities (moderate, severe or very severe) due to the limited number of field samples in each category.

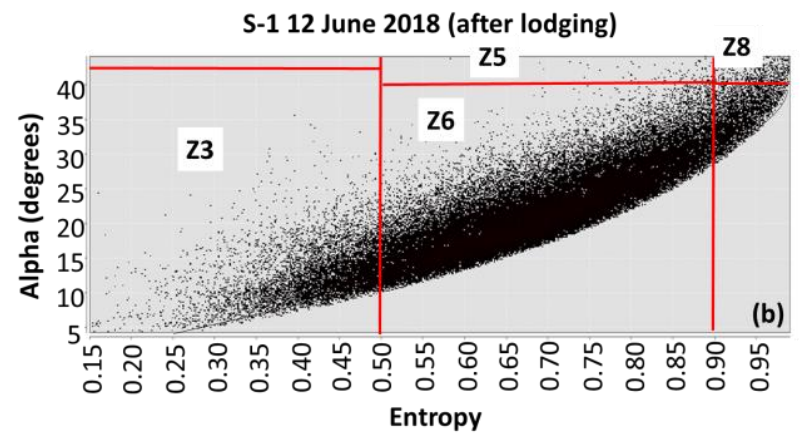

R-2 FQ8 13 June 2018 (after lodging)

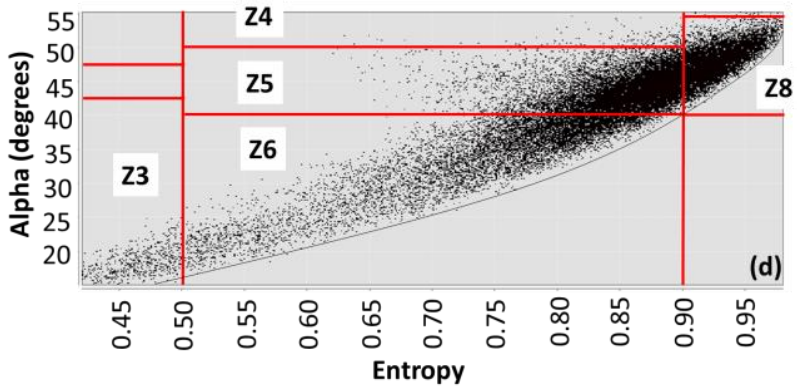

Figure 3. H- $\alpha$ classification planes from before and after lodging image pairs derived using near date (a), (b) Sentinel-1 (25 April, 12 June) and (c), (d) low incidence angle RADARSAT-2 FQ8 (26 April, 13 June) data.

\begin{tabular}{|c|c|c|c|c|c|}
\hline Zones & Description & \multicolumn{4}{|c|}{ Percentage scattering } \\
\hline & & $\begin{array}{c}\text { S-1 } \\
\text { [25 Apr] } \\
\text { (before) }\end{array}$ & $\begin{array}{c}\text { S-1 } \\
\text { [12 Jun] (after) }\end{array}$ & $\begin{array}{c}\text { R-2 FQ8 } \\
\text { [26 Apr] (before) }\end{array}$ & $\begin{array}{c}\text { R-2 FQ8 } \\
\text { [13 Jun] } \\
\text { (after) }\end{array}$ \\
\hline $\mathrm{Z} 1$ & Dihedral reflector & $0.00 \%$ & $0.00 \%$ & $0.00 \%$ & $0.00 \%$ \\
\hline $\mathrm{Z} 2$ & Dipole & $0.00 \%$ & $0.00 \%$ & $0.00 \%$ & $0.00 \%$ \\
\hline $\mathrm{Z3}$ & Bragg surface & $6.11 \%$ & $12.87 \%$ & $7.02 \%$ & $8.79 \%$ \\
\hline $\mathrm{Z} 4$ & Double reflection & $2.29 \%$ & $0.00 \%$ & $19.63 \%$ & $8.28 \%$ \\
\hline $\mathrm{Z5}$ & Anisotropic particles & $12.78 \%$ & $22.19 \%$ & $24.01 \%$ & $25.76 \%$ \\
\hline Z6 & Random surface & $41.01 \%$ & $39.51 \%$ & $25.03 \%$ & $27.00 \%$ \\
\hline $\mathrm{Z7}$ & Complex structures & $0.00 \%$ & $0.00 \%$ & $0.00 \%$ & $10.23 \%$ \\
\hline $\mathrm{Z} 8$ & $\begin{array}{c}\text { Random anisotropic } \\
\text { scattering }\end{array}$ & $9.37 \%$ & $7.40 \%$ & $24.31 \%$ & $19.94 \%$ \\
\hline Z9 & Non-feasible & $28.43 \%$ & $18.03 \%$ & $0.00 \%$ & $0.00 \%$ \\
\hline decrease & & & & & \\
\hline Increase & & & & & \\
\hline
\end{tabular}

Table 2. Percentage scattering in each zone from before and after lodging image pairs derived using near date Sentinel-1 and low incidence angle RADARSAT-2 FQ8 data. Main scattering mechanism changes are highlighted in the table. The main one is in bold. 
S-1 25 April 2018 (before lodging)

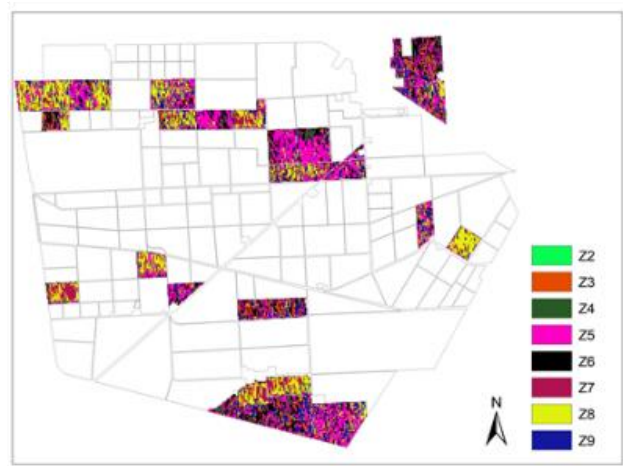

R-2 FQ8 26 April 2018 (before lodging)
S-1 12 June 2018 (after lodging)

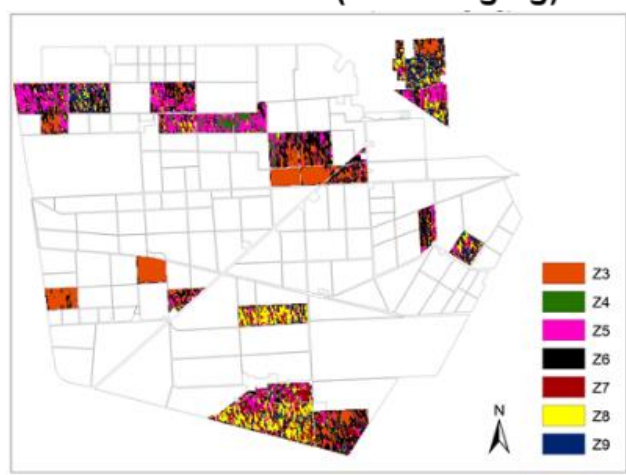

R-2 FQ8 13 June 2018 (after lodging)

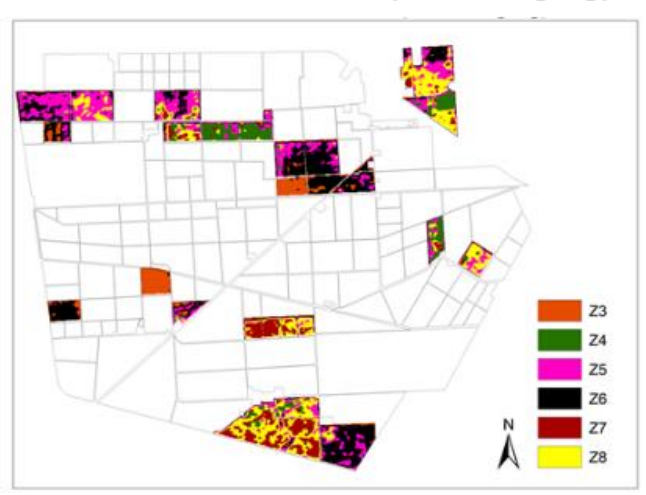

Figure 4. Combined $\mathrm{H} / \alpha$ and Wishart unsupervised classification of scattering mechanisms from before and after lodging image pairs derived using near date Sentinel-1 (25 April, 12 June) and low incidence angle RADARSAT-2 FQ8 (26 April, 13 June) data.

"RADARSAT-2 Data and Products. MacDonald, Dettwiler and Associates Ltd. (2018) - All Rights Reserved. RADARSAT is an official trademark of the Canadian Space Agency."

\subsection{Assessment of scattering mechanisms using S-1 and high incidence angle R-2 FQ21}

The same date satellite image pairs of S-1 and R-2 FQ21 were used to facilitate the comparison of the two sensors. Fig. 5 depicts the scatter plots for each image in the $\mathrm{H}-\alpha$ classification plane, while the quantitative percentage statistics of the scattering mechanisms from the healthy and lodged plots in each zone are shown in Table 3. The analysis of the distributions in Fig. 5 and the percentage scattering in Table 3 show that the $\mathrm{H} / \alpha$ values changed after lodging incidence. In the case of $\mathrm{S}-1$, the largest densities in the two planes correspond to surface scattering (Z6: $34.57 \%$ before and $46.09 \%$ after) with moderate to high randomness $(\mathrm{H})$ while the double bounce scattering (Z4) is non-existent. Even for high incidence R-2 FQ21 data, the largest densities in the two planes correspond to surface scattering (Z6: $19.62 \%$ before and $14.54 \%$ after) and volume diffusion (Z8: $11.62 \%$ before and $25.56 \%$ after) with moderate to high randomness with some double-bounce scattering (Z4: $14.29 \%$ before and $13.67 \%$ after) at moderate $\mathrm{H}$.

The change in the scattering mechanisms was found to be less consistent across S-1 and R-2 FQ21 data in most of the zones (Table 3) unlike with low incidence R-2 data. The low entropy Z2 volume scattering decreased by $11.2 \%$ after lodging at high incidence angle in R-2 data while there was no change in S-1 data (Table 3). Unlike R-2 FQ8, Z3 Bragg scattering decreased moderately after lodging with both S-1 and R-2 FQ21 datasets (Table 3). At high incidence angle, radar signatures of cereal crop canopies are dominated by the volume scattering (Z6) component due to longer path length within the canopy while the surface (Bragg) scattering (Z3) is reduced. A contrasting change in the moderate entropy Z5 and Z6 scattering was observed between the S-1 and R-2 FQ21 data. With S-1 data, $\mathrm{Z} 5$ scattering reduced by $9 \%$ while in the latter case, it increased after lodging (Table 3). Z5 scattering is the scattering caused by a volume of anisotropic particles and indicates the presence of multiple scatterers. It is expected that in a lodged crop, the presence of highly random anisotropic scattering elements would increase, causing an increase in Z5 scattering as shown in the case of R-2 data.

Furthermore, Z6 scattering increased with lodging (11.5\%) in S1 data while with R-2 FQ21 data, it reduced (Table 3). Surface scattering adds to the Z6 scattering but with moderate entropy, and as discussed earlier surface scattering reduces at high incidence angles. Lastly, the high entropy random anisotropic scattering (Z8) increased by $14 \%$ with high incidence R-2 data, after the crop lodged. This can be explained by the highly random distribution of lodged canopy in different directions.

However, it is important to note that in S-1 and R-2 FQ8 analysis, the changes in crop condition between the period of April to June were more extreme, with more cases of lodging at the end of the season unlike with S-1 and R-2 FQ21. Even though S-1 and R-2 FQ21 have similar incidence angles, the change in scattering mechanisms was still evident which can be attributed to different polarization modes (dual/quad) and spatial resolution $(15 \mathrm{~m} / 7 \mathrm{~m})$. The classified $\mathrm{S}-1$ and R-2 image pairs are shown in Fig. 6. The unsupervised classification again 
allowed the identification of various scattering mechanisms and helped discriminate the scattering mechanisms for healthy and lodged classes in general. However, like S-1 and R2 FQ8 datasets, no unique scattering mechanism could be associated with different lodging severities (moderate, severe or very severe) due to the limited number of field samples in each category. To extract the unique scattering mechanisms for different lodging classes, in addition to a sufficient number of samples, it would be important to consider more polarimetric features in a theoretical modelling approach based on radiative transfer theory). This approach could be (very complex and was beyond the scope of this study.
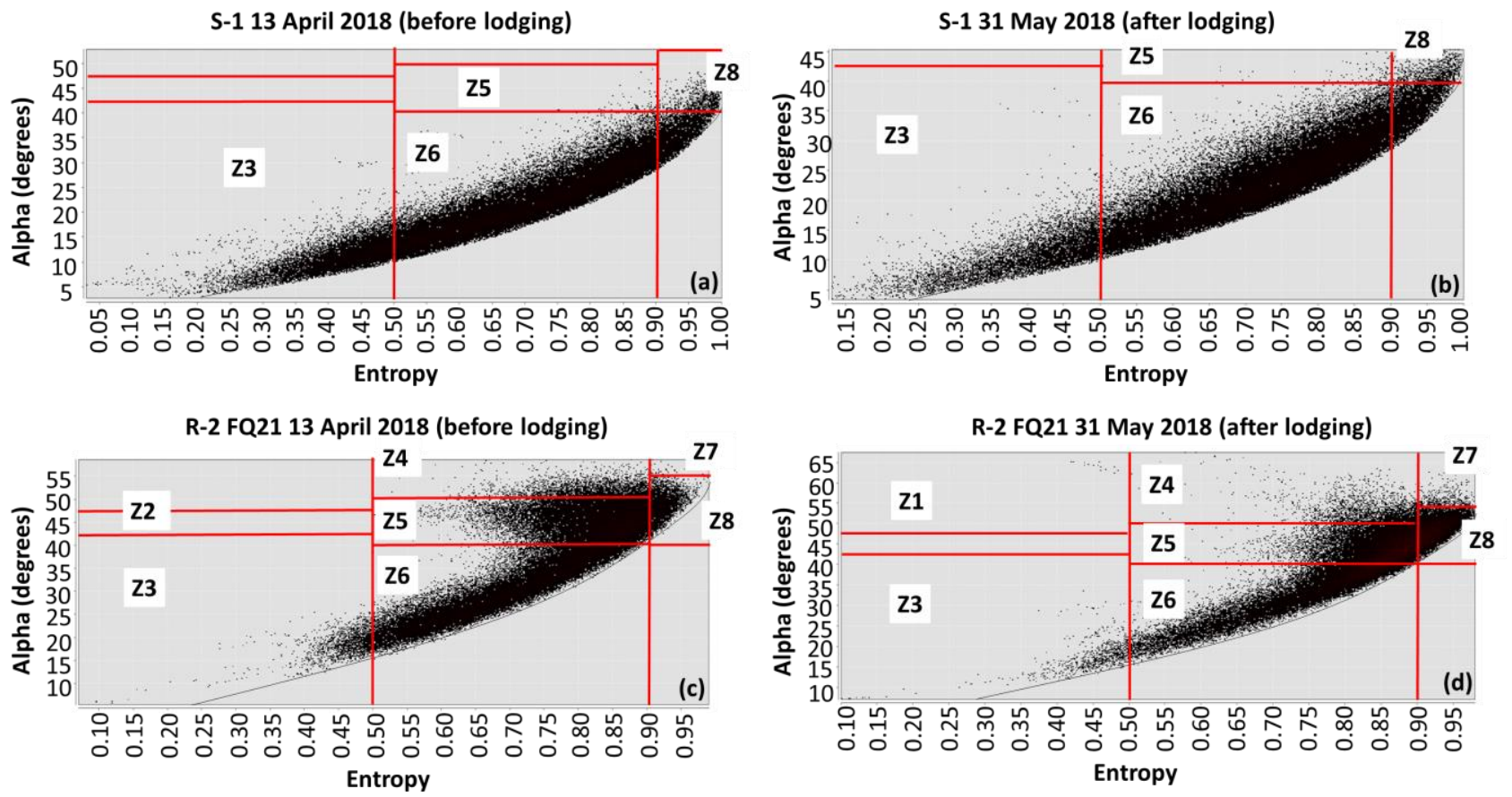

Figure 5. H- $\alpha$ classification planes from before and after lodging image pairs derived using same date (a), (b) Sentinel-1 (13 April, 31 May) and (c), (d) high incidence angle RADARSAT-2 FQ21 (13 April, 31 May) data

\begin{tabular}{|c|c|c|c|c|c|}
\hline Zones & Description & \multicolumn{3}{|c|}{ Percentage scattering } \\
\hline & & $\begin{array}{c}\text { S-1 } \\
{[\mathbf{1 3} \text { Apr] }} \\
\text { (before) }\end{array}$ & $\begin{array}{c}\text { S-1 } \\
{[\mathbf{3 1} \text { May] }} \\
\text { (after) }\end{array}$ & $\begin{array}{c}\text { R-2 FQ8 } \\
{[\mathbf{1 3} \text { Apr] }} \\
\text { (before) }\end{array}$ & $\begin{array}{c}\text { R-2 FQ8 } \\
\text { [31 May] } \\
\text { (after) }\end{array}$ \\
\hline Z1 & Dihedral reflector & $0.00 \%$ & $0.00 \%$ & $0.00 \%$ & $0.31 \%$ \\
\hline $\mathbf{Z 2}$ & Dipole & $0.00 \%$ & $0.00 \%$ & $11.17 \%$ & $0.00 \%$ \\
\hline $\mathbf{Z 3}$ & Bragg surface & $12.10 \%$ & $3.42 \%$ & $14.34 \%$ & $9.39 \%$ \\
\hline $\mathbf{Z 4}$ & Double reflection & $0.00 \%$ & $0.00 \%$ & $14.29 \%$ & $13.67 \%$ \\
\hline $\mathbf{Z 5}$ & Anisotropic particles & $18.68 \%$ & $9.74 \%$ & $17.40 \%$ & $20.56 \%$ \\
\hline $\mathbf{Z 6}$ & Random surface & $34.57 \%$ & $46.10 \%$ & $19.62 \%$ & $14.54 \%$ \\
\hline $\mathbf{Z 7}$ & Complex structures & $0.00 \%$ & $0.00 \%$ & $11.57 \%$ & $15.97 \%$ \\
\hline $\mathbf{Z 8}$ & Random anisotropic scattering & $11.54 \%$ & $8.11 \%$ & $11.62 \%$ & $25.56 \%$ \\
\hline Z9 & Non-feasible & $23.13 \%$ & $32.64 \%$ & $0.00 \%$ & $0.00 \%$ \\
\hline decrease & & & & & \\
\hline Increase & & & & & \\
\hline
\end{tabular}

Table 3. Percentage scattering in each zone from before and after lodging image pairs derived using same date Sentinel-1 and high incidence angle RADARSAT-2 FQ21 data. 
S-1 13 April 2018 (before lodging)

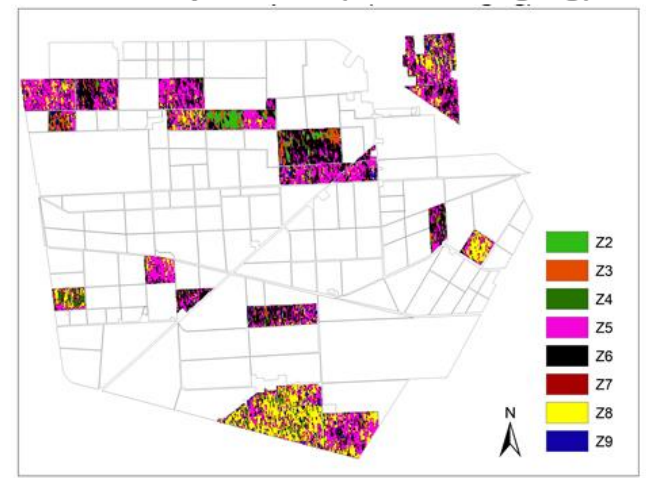

R-2 FQ21 13 April 2018 (before lodging)

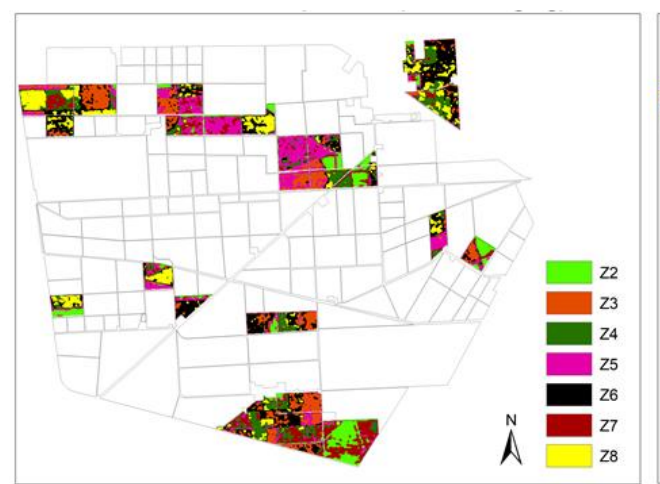

S-1 31 May 2018 (after lodging)



R-2 FQ21 31 May 2018 (after lodging)

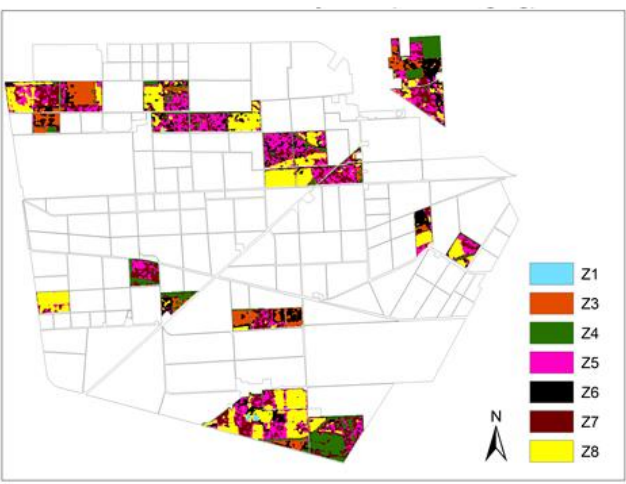

Figure 6. Combined $\mathrm{H} / \alpha$ and Wishart unsupervised classification of scattering mechanisms from before and after lodging image pairs derived using same date Sentinel-1 (13 April, 31 May) and high incidence angle RADARSAT-2 FQ21 (13 April, 31 May) data,

"RADARSAT-2 Data and Products. MacDonald, Dettwiler and Associates Ltd. (2018) - All Rights Reserved. RADARSAT is an official trademark of the Canadian Space Agency."

\section{CONCLUSIONS}

This study demonstrated the feasibility of using SAR data to detect the possible change in scattering mechanisms in the event of lodging in wheat; thereby allowing lodging identification to support precision management and understanding of on-going plant physiological processes. Even though a few studies have shown the potential of SAR data for crop lodging assessment, knowledge regarding the possible change in SAR signatures (as a function of scattering mechanisms) was lacking in the literature, and this study aimed to fill this gap. Pairs of dual-pol Sentinel-1 and multi-incidence angle quad-pol RADARSAT-2 data were examined to attain the objective. The scattering mechanisms in nine zones of the $\mathrm{H}-\alpha$ space were extracted and compared from before and after lodging image pairs using an unsupervised $\mathrm{H} / \alpha$ decomposition and Wishart classifier.

Overall, we found consistent changes in scattering mechanisms between S-1 and low incidence angle R-2 FQ8 data while with high incidence angle R-2 FQ21 data, the changes were inconsistent in most cases. We also found a contrast in the change in scattering mechanisms between low and high incidence angle R-2 data. The Bragg scattering (Z3) increased after lodging at low incidence angle $(1.8 \%)$ while at high incidence angle, $\mathrm{Z} 3$ showed a decreasing trend (8\%). The Z4 scattering which corresponds to double bounce scattering at high entropy decreased after lodging at low incidence angle while at high incidence angle, the change was negligible. The high incidence angle $\mathrm{Z7}$ and $\mathrm{Z} 8$ scattering increased after lodging, confirming the random anisotropic nature of the lodged canopy. The contrasting behaviour of Sentinel-1 and RADARSAT- 2 data in some cases can be due to differences in the configuration of the two sensors.

These results underline how SAR-derived metrics can advance conventional lodging evaluation methods. Although we believe that these results provide basic insights into the dominant scattering mechanisms during lodging, further research is required with more image pairs along with more sophisticated modelling approaches to assess significant changes in scattering after lodging. Further efforts can also be directed towards incorporating the dielectric properties of the healthy and lodged crops to provide a wider variation in backscatter values. Correlation and regression methods with different degrees of lodging severities can be tested to provide more robust quantitative estimates.

\section{ACKNOWLEDGEMENTS}

The authors thank all those who actively participated in the field campaign in 2018. We are grateful to Dr Donato Cillis of IBF-S technical team for his support and the Bonifiche Ferraresi farm for hosting the experimentation and for supporting the field activities for the period 2017-2018. The authors also thank MDA-GSI and the Canadian Government for providing RADARSAT-2 data through the project "Vegetation parameter retrieval from SAR data", number SOAR-EI-5446. 


\section{REFERENCES}

Balenzano, A., Mattia, F., Satalino, G., Davidson, M.W.J., 2010. Dense temporal series of C-and L-band SAR data for soil moisture retrieval over agricultural crops. IEEE J. Sel. Top. Appl. Earth Obs. Remote Sens. 4, 439-450.

Berry, P.M., Sterling, M., Spink, J.H., Baker, C.J., SylvesterBradley, R., Mooney, S.J., Tams, A.R., Ennos, A.R., 2004. Understanding and reducing lodging in cereals. Adv. Agron. 84, 215-269.

Chapman, S., Merz, T., Chan, A., Jackway, P., Hrabar, S., Dreccer, M., Holland, E., Zheng, B., Ling, T., JimenezBerni, J., 2014. Pheno-Copter: A low-altitude, autonomous remote-sensing robotic helicopter for highthroughput field-based phenotyping. Agronomy 4, 279 301.

Chauhan, S., Darvishzadeh, R., Boschetti, M., Nelson, A., 2020a. Estimation of crop angle of inclination for lodged wheat using multi-sensor SAR data. Remote Sens. Environ. 236, 111488.

Chauhan, S., Darvishzadeh, R., Boschetti, M., Nelson, A., 2020b. Understanding Wheat Lodging using MultiTemporal Sentinel-1 and Sentinel-2 Data. Remote Sens. Environ. 243, 111804.

Chen, J., Li, H., Han, Y., 2016. Potential of RADARSAT-2 data on identifying sugarcane lodging caused by typhoon. 5th Int. Conf. Agro-Geoinformatics, Agro-Geoinformatics 16.

Chen, S.-W., Wang, X.-S., Xiao, S.-P., Sato, M., 2013. General polarimetric model-based decomposition for coherency matrix. IEEE Trans. Geosci. Remote Sens. 52, $1843-$ 1855.

Cloude, S.R., Pottier, E., 1997. An entropy based classification scheme for land applications of polarimetric SAR. IEEE Trans. Geosci. Remote Sens. 35, 68-78.

Gerten, D.M., Wiese, M.V., 1987. Microcomputer-assisted video image analysis of lodging in winter wheat. Photogramm. Eng. Remote Sens. 53, 83-88.

Guo, J., Wei, P.-L., Liu, J., Jin, B., Su, B.-F., Zhou, Z.-S., 2018. Crop classification based on differential characteristics of H/Alpha scattering parameters for multitemporal quadand dual-polarization SAR images. IEEE Trans. Geosci. Remote Sens. 56, 6111-6123.

Jafari, M., Maghsoudi, Y., Zoej, M.J.V., 2015. A new method for land cover characterization and classification of polarimetric SAR data using polarimetric signatures. IEEE J. Sel. Top. Appl. Earth Obs. Remote Sens. 8, 3595-3607.

Jagdhuber, T., Stockamp, J., Hajnsek, I., Ludwig, R., 2014. Identification of soil freezing and thawing states using SAR polarimetry at C-band. Remote Sens. 6, 2008-2023.

Lee, J.-S., Ainsworth, T.L., Wang, Y., 2013. Generalized polarimetric model-based decompositions using incoherent scattering models. IEEE Trans. Geosci. Remote Sens. 52, 2474-2491.

Lee, J.-S., Grunes, M.R., Ainsworth, T.L., Du, L.-J., Schuler, D.L., Cloude, S.R., 1999. Unsupervised classification using polarimetric decomposition and the complex Wishart classifier. IEEE Trans. Geosci. Remote Sens. 37, 2249-2258.

Łoś, H., Pawlowski, B., Osińska-Skotak, K., PlutoKossakowska, J., 2016. Comparison of quad-polarimetric and dual-polarimetric SAR data capabilities for river Ice classification, in: Living Planet Symposium. pp. 331335.

Peng, D., Chen, X., Yin, Y., Lu, K., Yang, W., Tang, Y., Wang, Z., 2014. Lodging resistance of winter wheat (Triticum aestivum L.): Lignin accumulation and its related enzymes activities due to the application of paclobutrazol or gibberellin acid. F. Crop. Res. 157, 1-7.

Pinthus, M.J., 1974. Lodging in wheat, barley, and oats: the phenomenon, its causes, and preventive measures. Adv. Agron. 25, 209-263.

Shu, M., Zhou, L., Gu, X., Ma, Y., Sun, Q., Yang, G., Zhou, C., 2019. Monitoring of maize lodging using multi-temporal Sentinel-1 SAR data. Adv. Sp. Res. 65, 470-480.

Wang, H., Magagi, R., Goita, K., Jagdhuber, T., Hajnsek, I., 2016. Evaluation of simplified polarimetric decomposition for soil moisture retrieval over vegetated agricultural fields. Remote Sens. 8, 142.

Yang, H., Chen, E., Li, Z., Zhao, C., Yang, G., Pignatti, S., Casa, R., Zhao, L., 2015. Wheat lodging monitoring using polarimetric index from RADARSAT-2 data. Int. J. Appl. Earth Obs. Geoinf. 34, 157-166.

Yang, M.D., Huang, K.S., Kuo, Y.H., Tsai, H., Lin, L.M., 2017. Spatial and spectral hybrid image classification for rice lodging assessment through UAV imagery. Remote Sens. $9,583$. 